\title{
Search for the H Chondrite Parent Body among the Three Largest S-type Asteroids: (3) Juno, (7) Iris, and (25) Phocaea
}

\author{
John W. Noonan ${ }^{1}$ (D), Vishnu Reddy ${ }^{1}$ (D), Walter M. Harris ${ }^{1}$ (D), William F. Bottke ${ }^{2}$, Juan A. Sanchez ${ }^{3}$, Roberto Furfaro ${ }^{4}$, \\ Zarah Brown ${ }^{1}$, Rachel Fernandes ${ }^{1}$, Theodore Kareta ${ }^{1}$ (D), Cassandra Lejoly ${ }^{1}$, Ravi Teja Nallapu ${ }^{1}$, Haris Khan Niazi ${ }^{1}$, \\ Lindsay R. Slick ${ }^{1}$, Lauren Schatz ${ }^{5}$, Benjamin N. L. Sharkey ${ }^{1}$, Alessondra Springmann ${ }^{1}$, Geoff Angle ${ }^{1}$, Leandra Bailey ${ }^{1}$, \\ Derian D. Acuna ${ }^{1}$, Collin Lewin ${ }^{1}$, Katherina Marchese ${ }^{1}$, Max Meshel ${ }^{1}$, Natalie Quintero ${ }^{1}$, Kyle Tatum ${ }^{1}$, and Greg Wilburn ${ }^{1}$ \\ ${ }^{1}$ Lunar and Planetary Laboratory, University of Arizona, 1629 E. University Boulevard, Tucson, AZ 85721-0092, USA; noonan@lpl.arizona.edu \\ ${ }^{2}$ Southwest Research Institute, Suite 3001050 Walnut Street, Boulder, CO 80301, USA \\ ${ }^{3}$ Planetary Science Institute, 1700 E. Fort Lowell Road, Tucson, AZ 85719, USA \\ ${ }^{4}$ Department of Systems and Industrial Engineering, University of Arizona, 1127 E. James E. Rogers Way, Tucson, AZ 85721-0020, USA \\ ${ }^{5}$ College of Optical Sciences, University of Arizona, 1630 E. University Boulevard, Tucson, AZ 85721-0092, USA \\ Received 2018 December 19; revised 2019 September 23; accepted 2019 September 24; published 2019 November 1
}

\begin{abstract}
Linking meteorites to source regions in the main asteroid belt is important for understanding the conditions under which their parent bodies formed. Ordinary chondrites are the most abundant class of meteorites on Earth, totaling $86 \%$ of all collected samples. Some S-type asteroids/families have been proposed as sources for the three different (H, L, and LL) types of ordinary chondrites with Hebe, Agnia, Merxia, and Koronis families being the source for $\mathrm{H}$ chondrites, Gefion for $\mathrm{H} / \mathrm{L}$ chondrites, and Flora family for LL chondrites. However, the composition and meteorite affinity of several large S-type main belt asteroids remains unconstrained leaving the possibility of additional source regions for ordinary chondrite meteorites. Here we investigate the surface composition of three large S-type asteroids, (3) Juno, (7) Iris, and (25) Phocaea, using their near-infrared spectra $(0.7-2.55 \mu \mathrm{m})$ to identify the parent body of the $\mathrm{H}$ chondrites. We use a Bayesian inference model to confirm the meteorite analogs of the three asteroids. Based on our Bayes classifier we find the following analogs and probabilities: Juno is likely $\mathrm{H}$ chondrite $(89 \%)$, Iris is likely LL chondrite $(97.5 \%)$, and Phocaea is likely H chondrite (98.6\%). While Phocaea has the highest probability of being an $\mathrm{H}$ chondrite, it is dynamically unlikely to deliver material to near-Earth space. While Juno has spectral properties similar to $\mathrm{H}$ chondrites, its family is unlikely to produce sizeable $\mathrm{H}$-chondrite-type near-Earth objects (NEOs). If Juno is the primary source of $\mathrm{H}$ chondrite meteorites, it suggests that an additional source is needed to explain the H-chondrite-type NEOs.
\end{abstract}

Key words: infrared: planetary systems - minor planets, asteroids: individual (Juno, Iris, Phocaea) - techniques: spectroscopic

\section{Introduction}

Identifying source regions from which near-Earth asteroids (NEAs) and meteorites originate provides key constraints on the conditions in the solar nebula under which their parent bodies formed and subsequently evolved. Remote sensing in the form of near-infrared (NIR) spectroscopy $(0.7-2.5 \mu \mathrm{m})$ is the best method to link these three populations (meteorites, NEAs, and main belt asteroids). S-type asteroids are characterized by moderate surface albedos and silicate absorption features due to the minerals olivine and pyroxene (Masiero et al. 2011). A subset of the S-type asteroids has been proposed to be the parent bodies of ordinary chondrite meteorites (Gaffey et al. 1993). Ordinary chondrites are the most common type and make up $86 \%$ of all meteorites in terrestrial collections (Meteoritical Bulletin). They are classified under three chemically/isotopically distinct chondrite groups, H, L, and LL chondrites (i.e., high, low, and very low iron content, respectively), originating from different parent bodies.

Several asteroids and their families have been proposed as sources of the ordinary chondrites. Gaffey \& Gilbert (1998) proposed (6) Hebe as a probable parent body of the $\mathrm{H}$ chondrites, but the asteroid lacks a family capable of delivering the meteorites (Nesvorný 2015). Nesvorný et al. (2009) and Vernazza et al. (2014) have proposed the Gefion asteroid family as the source region of $\mathrm{L}$ chondrites. But more recent observations by McGraw et al. (2018) show that Gefion might have a mixture of $\mathrm{H}$ and $\mathrm{L}$ chondrites. The Flora family in the inner main belt has been proposed as the source family of the LL chondrites (Vernazza et al. 2008, 2014).

Of these three asteroid-meteorite links, the LL-chondriteFlora link is the most robust. The source families for $\mathrm{L}$ and $\mathrm{H}$ chondrites remains ambiguous although several families have been proposed as possible sources by Vernazza et al. (2014) including Agnia, Merxia, and Koronis families for $\mathrm{H}$ chondrites and Gefion for $\mathrm{H} / \mathrm{L}$ chondrites. Previous asteroid-meteorite link papers have focused on either large groups of unrelated main belt asteroids (Gaffey et al. 1993) or specific families (Vernazza et al. 2008). However, in this paper we investigate the surface composition of three large S-type main belt asteroids (3) Juno, (7) Iris, and (25) Phocaea to identify their affinity to ordinary chondrite meteorites. While Juno and Phocaea have associated asteroid families, no families have been identified for Iris (Masiero et al. 2015). In this paper we specifically focused on finding the source family for $\mathrm{H}$ chondrites.

Constraining the source of the $\mathrm{H}$ chondrites to one or more of these asteroids would provide several key pieces of information that would influence the meteorites' scientific interpretation. If a family or parent body can be identified as a single source, inferences about the conditions that the parent body formed in can be made. Additionally, if a source can be 
Table 1

Asteroid Characteristics

\begin{tabular}{|c|c|c|c|c|c|c|}
\hline Asteroid & Dimensions & Period & Albedo & Density & Taxonomic Type & Misc. \\
\hline (3) Juno & $320 \times 267 \times 200 \pm 6 \mathrm{~km}(\mathrm{a}),(\mathrm{b}),(\mathrm{c})$ & $\begin{array}{l}7.21 \mathrm{hr} \\
\text { (d), (e) }\end{array}$ & 0.205 (e) & $\begin{array}{c}3.32 \\
\mathrm{~g} \mathrm{~cm}^{3}(\mathrm{c})\end{array}$ & Sq (f) S(IV) (g) & $\begin{array}{l}\text { Possible } 100 \text { km. cra- } \\
\text { ter (a) }\end{array}$ \\
\hline (7) Iris & $225 \times 190 \times 190 \mathrm{~km}(\mathrm{~h})$ & $7.14(\mathrm{e})$ & 0.277 (i) & $\mathrm{N} / \mathrm{A}$ & Sk (f) S(IV) (g) & $\begin{array}{l}\text { Significant albedo varia- } \\
\text { tions }(\mathrm{j})\end{array}$ \\
\hline (25) Phocaea & 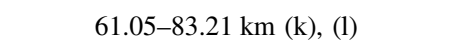 & $9.93 \mathrm{hr}(\mathrm{m})$ & $0.189-0.350(\mathrm{k}),(\mathrm{l})$ & $\mathrm{N} / \mathrm{A}$ & $\mathrm{S}(\mathrm{IV})(\mathrm{g})$ & $\mathrm{N} / \mathrm{A}$ \\
\hline
\end{tabular}

Note. (a) Baliunas et al. (2003), (b) Ďurech et al. (2011), (c) Viikinkoski et al. (2015), (d) Groeneveld \& Kuiper (1954), (e) Warner et al. (2009), (f) DeMeo et al. (2009), (g) Gaffey et al. (1993), (h) Kaasalainen et al. (2002), (i) Tedesco et al. (2004), (j) Conrad et al. (2003), (k) Usui et al. (2011), (1) Masiero et al. (2012), (m) Pilcher (2011).

identified the dynamic, thermal, and collisional histories of the meteorites and their journey from their source can be studied via modeling. Given that $34 \%$ of meteorites that have fallen to Earth are $\mathrm{H}$ chondrites it may also be the case that there are multiple sources as well, but it is still critical to assess possible candidates and our current knowledge of them. A brief summary of the physical characteristics of our targets is presented in Table 1.

(3) Juno is the second largest S-type asteroid and has a proper semimajor axis, eccentricity, and inclination of $2.669 \mathrm{au}$, 0.257 , and 12.99 , respectively (Figure 1). Ground-based observations carried out by Viikinkoski et al. (2015) showed that Juno's shape is irregular and may include a possible $100 \mathrm{~km}$ crater (Baliunas et al. 2003). Comparing the $3.32 \mathrm{~g} \mathrm{~cm}^{3}$ density of Juno with that of L ordinary chondrites, Viikinkoski et al. (2015) suggest a porosity of 7\%, consistent with an intact interior structure. Radar observations of Juno have shown that it has moderate decimeter-scale near-surface structure, and moderate radar reflectivity, consistent with other S-type objects (Magri et al. 2007). Juno is classified as an Sq-class asteroid in the S complex by DeMeo et al. (2009), and S(IV) in the Gaffey et al. (1993) scheme. Based on these classifications, Juno's surface is thought to be similar to ordinary chondrites. The combination of possible craters and an ordinary chondrite-like surface composition makes the asteroid a possible meteorite source.

Modeling by Nesvorný (2015) has shown that the Juno asteroid family consists of at least 1683 members. The largest of these, (32326) 2000 Q0062, is $6 \mathrm{~km}$ in diameter with an albedo and spectral class similar to Juno. However, analysis by Oszkiewicz et al. (2012) using photometric phase functions and their correlation to asteroid taxonomy showed no clear dominant taxonomical type within the Juno family.

Our second target, (7) Iris, is in the middle of the inner main belt (Figure 1). Of key interest are the combination of radar observations of (7) Iris that revealed large concavities on the southern hemisphere (Ostro et al. 2010) and spectral observations of Iris that match that of L chondrites (Ueda et al. 2003). These factors make (7) Iris a possible source of ordinary chondrites worth examining further.

Iris-produced meteoroids can escape the main belt via Yarkovsky thermal drift forces and resonances (e.g., Bottke et al. 2006), but the strength of Iris as a meteoroid source compared to other possibilities may be limited. Iris is relatively far from the $v 6$ and 3:1 resonances, two major resonances that likely deliver meteorites to Earth (e.g., Granvik et al. 2018). It is modestly close to the Mars 1:2 mean motion resonance and the Mars crossing zone, though the importance of each for

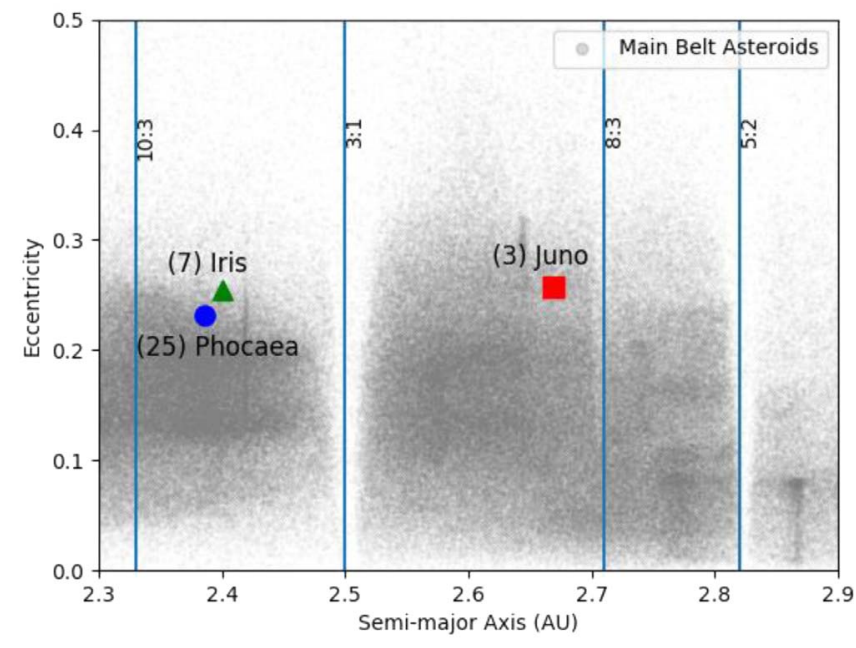

Figure 1. Eccentricity and semimajor axes for (3) Juno, (7) Iris, and (25) Phocaea plotted in comparison to other main belt asteroids and the most relevant Jupiter resonances.

meteorite delivery is not well quantified. There is no known asteroid family associated with Iris, so it presumably would not be as strong of a source of meteoroid material via a collisional cascade as a source with a family (e.g., Bottke et al. 2015). However, recent cratering events on Iris may produce steep size distributions of smaller fragments, with most of the material undetectable given current observation limits. The collision probability of asteroids with (7) Iris, a $200 \mathrm{~km}$ diameter body, is $3.47 \mathrm{e}-18 \mathrm{~km}^{-2} \mathrm{yr}^{-1}$ (e.g., Bottke et al. 1994). Here we assume that a $D>10 \mathrm{~km}$ body might produce enough small fragments to potentially be interesting. There are roughly 10,000 of them in the main belt (Bottke et al. 2005), so the mean interval between such impacts on Iris is 2.9 Gyr. This makes the probability of having such an event on Iris over the last $100 \mathrm{Myr}$ to be $0.1 \mathrm{Gyr} / 2.9 \mathrm{Gyr}=0.034=3.4 \%$. This unlikely scenario may represent the best likelihood for Iris to be a strong source of meteoroids.

The third asteroid we consider in this study is (25) Phocaea, located in the inner main belt at a relatively high inclination (Figure 1). Our study of Phocaea is motivated by the families linked to the asteroid. Analysis of the region near Phocaea has led to the discovery of two possible families. The Phocaea group, of which Phocaea is the largest, consists of both the Phocaea family of asteroids (Carruba 2009) and the Tamara family (Novaković et al. 2017). The Phocaea group is primarily comprised of S-type asteroids, while the Tamara family appears to be both $\mathrm{C}$ and $\mathrm{S}$ types. The Phocaea group is characterized by relatively high orbital inclination and eccentricity, as well as 
Table 2

Observing Circumstances

\begin{tabular}{|c|c|c|c|c|c|c|c|}
\hline Object & $\begin{array}{c}\text { Visual } \\
\text { Magnitude }\end{array}$ & $\begin{array}{l}\text { Heliocentric } \\
\text { Distance (au) }\end{array}$ & $\begin{array}{l}\text { Exposure } \\
\text { Time (s) }\end{array}$ & $\begin{array}{l}\text { Start } \\
\text { UT }\end{array}$ & Seeing (“) & $\begin{array}{c}\text { Phase } \\
\text { Angle }\left({ }^{\circ}\right)\end{array}$ & Airmass \\
\hline SAO162210 & 8.16 & $\mathrm{~N} / \mathrm{A}$ & 4 & $4: 32$ & 0.6 & $\mathrm{~N} / \mathrm{A}$ & 1.481 \\
\hline (3) Juno & 10.8 & 3.16 & 30 & $4: 38$ & 0.6 & 18 & 1.498 \\
\hline SAO162210 & 8.16 & $\mathrm{~N} / \mathrm{A}$ & 4 & $4: 49$ & 0.6 & $\mathrm{~N} / \mathrm{A}$ & 1.579 \\
\hline (3) Juno & 10.8 & 3.16 & 30 & $4: 55$ & 0.6 & 18 & 1.592 \\
\hline SAO162210 & 8.16 & $\mathrm{~N} / \mathrm{A}$ & 4 & $5: 05$ & 0.6 & $\mathrm{~N} / \mathrm{A}$ & 1.678 \\
\hline (3) Juno & 10.8 & 3.16 & 30 & $5: 11$ & 0.6 & 18 & 1.703 \\
\hline SAO162210 & 8.16 & $\mathrm{~N} / \mathrm{A}$ & 4 & $5: 23$ & 0.6 & $\mathrm{~N} / \mathrm{A}$ & 1.819 \\
\hline SAO74945 & 8.31 & $\mathrm{~N} / \mathrm{A}$ & 3 & $5: 39$ & 0.5 & $\mathrm{~N} / \mathrm{A}$ & 1.481 \\
\hline (7) Iris & 7.0 & 1.85 & 1 & $5: 45$ & 0.5 & 7 & 1.522 \\
\hline SAO74945 & 8.31 & $\mathrm{~N} / \mathrm{A}$ & 3 & $5: 49$ & 0.5 & $\mathrm{~N} / \mathrm{A}$ & 1.419 \\
\hline (7) Iris & 7.0 & 1.85 & 1 & $6: 01$ & 0.5 & 7 & 1.418 \\
\hline SAO74945 & 8.31 & $\mathrm{~N} / \mathrm{A}$ & 3 & $6: 05$ & 0.5 & $\mathrm{~N} / \mathrm{A}$ & 1.339 \\
\hline SAO 126941 & 9.54 & $\mathrm{~N} / \mathrm{A}$ & 10 & $6: 20$ & 0.5 & $\mathrm{~N} / \mathrm{A}$ & 1.11 \\
\hline (25) Phocaea & 11.4 & 1.48 & 60 & $6: 29$ & 0.5 & 30 & 1.152 \\
\hline (25) Phocaea & 11.4 & 1.48 & 45 & $6: 33$ & 0.5 & 30 & 1.61 \\
\hline SAO 126941 & 9.54 & $\mathrm{~N} / \mathrm{A}$ & 10 & 7:00 & 0.5 & $\mathrm{~N} / \mathrm{A}$ & 1.211 \\
\hline SAO74945 & 8.31 & $\mathrm{~N} / \mathrm{A}$ & 3 & $7: 11$ & 0.5 & $\mathrm{~N} / \mathrm{A}$ & 1.117 \\
\hline (7) Iris & 7.0 & 1.85 & 1.5 & $7: 19$ & 0.5 & 7 & 1.123 \\
\hline SAO74945 & 8.31 & $\mathrm{~N} / \mathrm{A}$ & 3 & $7: 25$ & 0.5 & $\mathrm{~N} / \mathrm{A}$ & 1.088 \\
\hline SAO93936 & 8.12 & $\mathrm{~N} / \mathrm{A}$ & 5 & $7: 33$ & 0.5 & $\mathrm{~N} / \mathrm{A}$ & 1.931 \\
\hline SAO93936 & 8.12 & $\mathrm{~N} / \mathrm{A}$ & 3 & 8:02 & 0.5 & $\mathrm{~N} / \mathrm{A}$ & 1.622 \\
\hline
\end{tabular}

its interaction with the secular $\left(\nu_{6}-\nu_{16}\right)$ resonance with Saturn (Carruba 2009). Most of this potential family's spectral classifications are S Class, with six exceptions that are not incongruous with the predicted interloper ratios from Migliorini et al. (1995).

The efficiency of high inclination bodies like the Phocaeas at producing meteorites has not been closely examined. Granvik et al. (2018) showed that the Phocaea region was only a minor source of near-Earth objects larger than $100 \mathrm{~m}$ in diameter (i.e., the order of a few percent). If we assume the situation for these bodies is also applicable to meteoroid-sized bodies, it seems likely that only a few of our ordinary chondrites come from this population.

\section{Observations}

NIR spectra $(0.7-2.5 \mu \mathrm{m})$ of Juno, Iris, and Phocaea were obtained using the SpeX instrument (Rayner et al. 2003) on the NASA Infrared Telescope Facility (IRTF) on Maunakea, Hawaii. Observations were made remotely on 2017 November 7 and the weather conditions were photometric with a seeing of 0 . $5-0$ ". 6 and relative humidity of $6 \%$ (Table 2). Spectra were acquired in prism mode with a resolution of $R \sim 200$ using a 0 ". 8 slit. Spectra were obtained using a nodding technique in which the object is alternated between two different slit positions ( $\mathrm{A}$ and $\mathrm{B}$ ) following the sequence ABBA. The slit was oriented along the parallactic angle during observations to minimize differential refraction at shorter wavelengths.

A description of the observations is provided here and is also available in Table 1. We acquired 30 individual spectra of Juno when the asteroid was at 10.8 visual magnitude at a heliocentric distance of $r=3.16$ au and a phase angle of $18^{\circ}$. Each exposure was limited to $30 \mathrm{~s}$ to prevent saturation. We also obtained 40 spectra of G2V-type local extinction star SAO 162210 before and after the asteroid observations to correct for telluric atmospheric features. For Iris, we obtained 50 spectra when the asteroid was at 7.0 visual magnitude at a heliocentric distance of $r=1.85$ au and a phase angle of $7^{\circ}$. Each exposure was limited to $1 \mathrm{~s}$. We obtained 50 spectra of G0-type local extinction star SAO 74945 spanning the Iris observations to correct for telluric atmospheric features. Finally, we obtained 22 spectra of asteroid Phocaea when it was at a visual magnitude of 11.4, a heliocentric distance of $r=1.48 \mathrm{au}$, and a phase angle of $30^{\circ}$. Each exposure was limited to $45 \mathrm{~s}$. We obtained 20 spectra of G1/2V-type local extinction star SAO 126941 surrounding the asteroid observations to correct for telluric atmospheric features. Finally, 30 spectra of solar analog star SAO 93936 were also obtained to correct for any spectral slope variation caused by the use of a non-solar extinction star.

Data reduction was performed using Spextool, a collection of IDL routines to perform wavelength calibration, telluric corrections, channel shifts, averaging, and display functions (Cushing et al. 2004). NASA IRTF provides the Spextool package for data reduction. A detailed description of the data reduction procedure is presented in Reddy et al. (2009) and Sanchez et al. (2013).

\section{Results}

NIR reflectance spectra of our three S-type asteroids, (3) Juno, (7) Iris, and (25) Phocaea, are presented in Figure 2. Spectra of all three asteroids show absorption bands centered at around 1 and $2 \mu \mathrm{m}$ indicating the presence of olivine and pyroxene, which are the typical characteristics of an S-type asteroid. Olivine has a composite band at $\sim 1 \mu \mathrm{m}$ (Band I) and no absorption band at $2 \mu \mathrm{m}$. Pyroxene has two absorption bands, one at $0.9 \mu \mathrm{m}$ (Band I) and another at $1.9 \mu \mathrm{m}$ (Band II). Spectral analysis is carried out by extracting diagnostic spectral band parameters such as band centers and a band area ratio (BAR). The band center is the reflectance minimum of a continuum-removed absorption feature and is diagnostic of mineral chemistry where the bands shift to longer wavelengths with increasing iron and/or calcium abundance. The ratio of the area of Band II to that of Band I is the BAR, which can be 


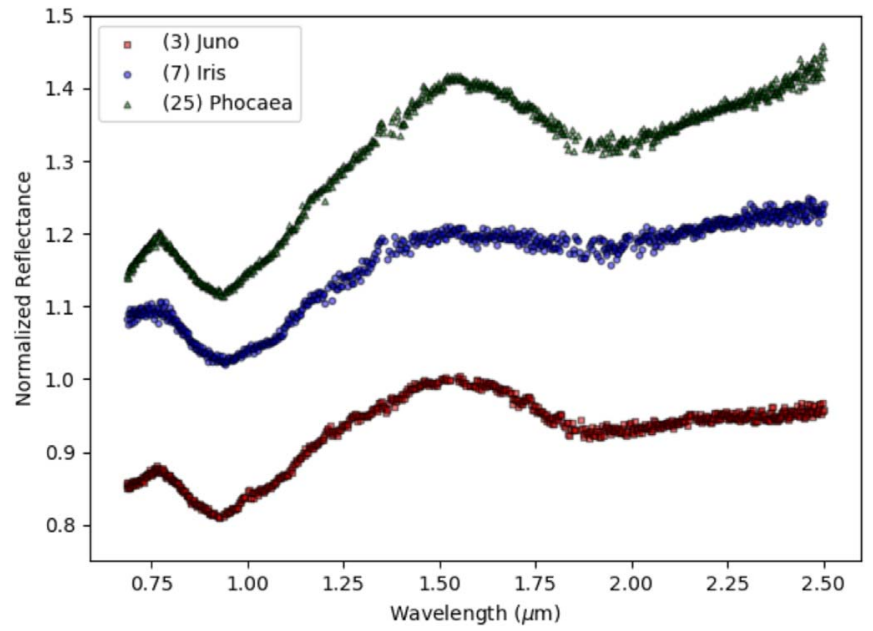

Figure 2. NIR spectrum of main belt asteroids (3) Juno, (7) Iris, and (25) Phocaea obtained with the SpeX instrument on the NASA IRTF. The spectra are plotted with an offset for clarity. All three asteroids show absorption bands at 1 (Band I) and $2 \mu \mathrm{m}$ (Band II) indicating the presence of the minerals olivine and pyroxene in their surface regolith.

used to constrain the relative abundance of olivine to pyroxene in ordinary chondrites.

For each asteroid spectrum the band centers and BARs were determined using a Python code similar to that used in Sanchez et al. (2015). Band centers were calculated after removing the continuum by fitting third- and fourth-order polynomials over the bottom third of each band. A total of 100 measurements were taken sampling a slightly different range of data points, and then averaged to obtain the final value. Band areas, which are defined as the areas between the continuum and the data curve, were measured using trapezoidal numerical integration and were used to calculate the BARs. Like the band centers, the final value is given by the average of 100 measurements. Uncertainties for these parameters correspond to the standard deviation of the mean.

Non-compositional effects such as temperature, grain size, and phase angle can alter spectral band parameters. For S(IV) asteroids, the BAR must be corrected for temperature-induced spectral effects before using it to infer surface composition. This results from differences between laboratory measurements made at room temperature and asteroid surface temperature. Such temperature corrections were derived from laboratory spectra of ordinary chondrites as described in Reddy et al. (2015). Spectral band parameters for the three S-type asteroids are shown in Table 3. Gaffey et al. (1993) used the Band I center versus BAR plot to identify subclasses of S-type asteroids with the polygonal region corresponding to the S(IV) subgroup having surface composition similar to ordinary chondrites; our Band I center versus BAR plot of the parameters is shown in Figure 3.

Cloutis et al. (1986) used the BAR to constrain the olivineto-pyroxene ratio in a mixture of those two minerals. Building upon this work, Dunn et al. (2010) applied the BAR to constrain the abundance of olivine to pyroxene in ordinary chondrite meteorites. Table 3 shows the BAR for the three $\mathrm{S}$-type asteroids we are investigating along with the olivine-to pyroxene ratio calculated using equations from Dunn et al. (2010). The equation of Dunn et al. (2010) was derived using equilibrated ordinary chondrite petrographic types (4-6) and matches the laboratory measured $\mathrm{x}$-ray diffraction (XRD)
Table 3

Asteroid Spectral Parameters

\begin{tabular}{lccc}
\hline \hline Object & $\begin{array}{c}\text { Band I Center } \\
(\mu \mathrm{m})\end{array}$ & BAR & ol(ol+px) \\
\hline (3) Juno & $0.932 \pm 0.009$ & $0.82 \pm 0.05$ & $0.53 \pm 0.03$ \\
(7) Iris & $0.954 \pm 0.008$ & $0.45 \pm 0.03$ & $0.62 \pm 0.03$ \\
(25) Phocaea & $0.945 \pm 0.008$ & $0.98 \pm 0.03$ & $0.49 \pm 0.03$ \\
\hline
\end{tabular}

Note. Band parameters and composition for the three asteroids. Uncertainties for the Band I center and BAR are given by the $1 \sigma$ error. The olivine-pyroxene abundance ratio $(\mathrm{ol} /(\mathrm{ol}+\mathrm{px}))$ was calculated using the equations of Dunn et al. (2010). Temperature corrections from Sanchez et al. (2012) were applied to the $\mathrm{BAR}$ and $\mathrm{ol} /(\mathrm{ol}+\mathrm{px})$.

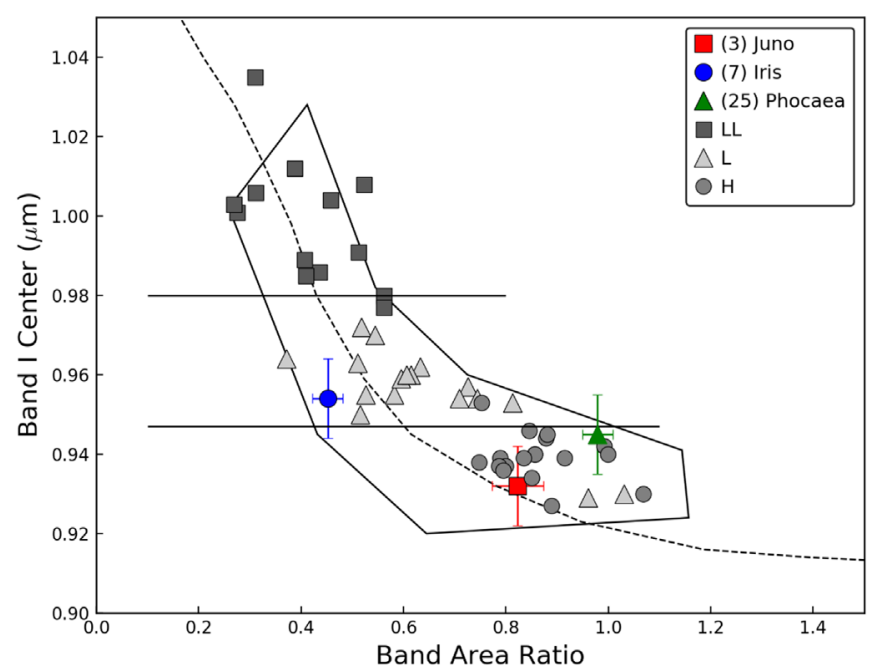

Figure 3. Band I center vs. BAR plot showing the location of our three main belt asteroids. Also shown are the values measured for LL, L, and H ordinary chondrites from Dunn et al. (2010). The polygonal region corresponds to the S(IV) subgroup of Gaffey et al. (1993). The dashed line indicates the location of the olivine-orthopyroxene mixing line of Cloutis et al. (1986). The plot shows that Juno falls in the $\mathrm{H}$ chondrite region, Phocaea in the transition zone between $\mathrm{H} / \mathrm{L}$ chondrites, and Iris in the L chondrite zone.

values as shown in Figure 4. Based on the BAR and the olivine-to-pyroxene ratio derived from it, (3) Juno plots between $\mathrm{H} / \mathrm{L}$ chondrites, (7) Iris plots with LL/L chondrites, and (25) Phocaea plots with $\mathrm{H} / \mathrm{L}$ chondrites in Figure 4. The $\mathrm{BAR}$ and olivine/pyroxene ratio derived meteorite analogs are broadly consistent with the Band I center versus BAR plot (Figure 3).

As a final step to confirm the meteorite analogs of our three asteroids we used the laboratory measured values of the BAR and $\mathrm{ol} /(\mathrm{ol}+\mathrm{px})$ of $\mathrm{H}, \mathrm{L}$, and LL for the ordinary chondrites to train a discriminative model capable of performing Bayesian inference by computing the posterior probability distribution given the meteoritic data (Reddy et al. 2018). The probability of the three classes (blue $=\mathrm{H}$, green $=\mathrm{L}$, and $\mathrm{R}=$ red $=\mathrm{LL}$ ) is highlighted in Figures 5 and 6. The color coded decision boundaries of the three classes as computed by the trained Bayes classifier are shown in Figure 5. The top panel of Figure 6 shows the posterior probability distribution computed by the classifier in the $(\mathrm{BAR}, \mathrm{ol} /(\mathrm{ol}+\mathrm{px}))$ space. The bottom panel of Figure 6 shows the contour plot representing the maximum value of the posterior probability as computed by the Bayes classifier. All plots reported in Figures 5 and 6 show the location of Iris, Juno, and Phocaea in the BAR, ol $/(\mathrm{ol}+\mathrm{px})$ space. 


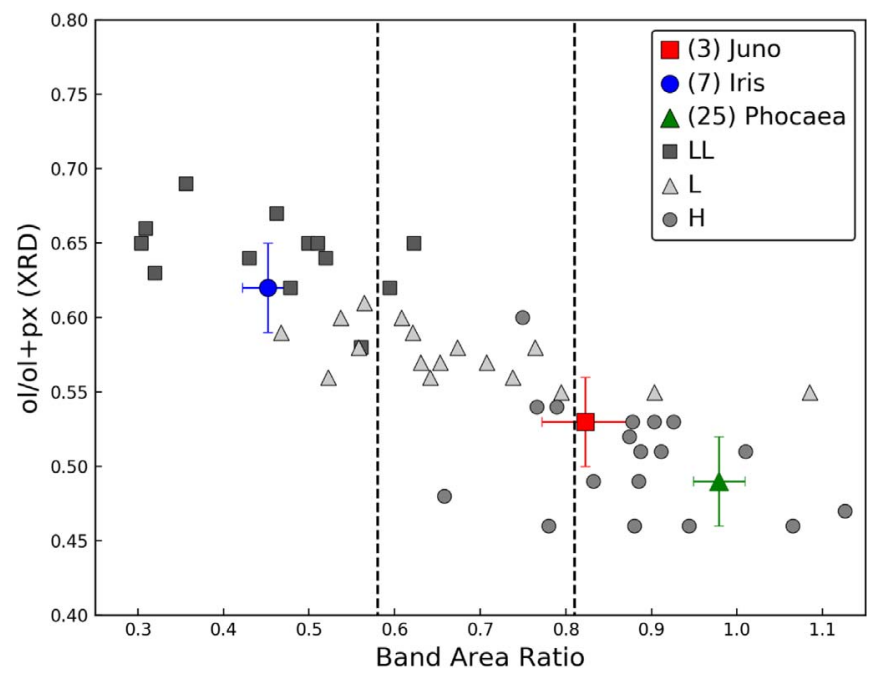

Figure 4. Olivine to pyroxene ratio vs band area ratio (BAR) for Juno, Iris, and Phocaea. The ol $/(\mathrm{ol}+\mathrm{px})$ ratio is derived using the method described in Dunn et al. (2010). Measured values for LL (squares), L (triangles), and H (circles) ordinary chondrites from Nakamura et al. (2011) are also included. The calculated values indicate that Juno and Phocaea's surface compositions are similar to $\mathrm{H} / \mathrm{L}$ chondrites while Iris bears more similarity to the $\mathrm{L} / \mathrm{LL}$ classification.

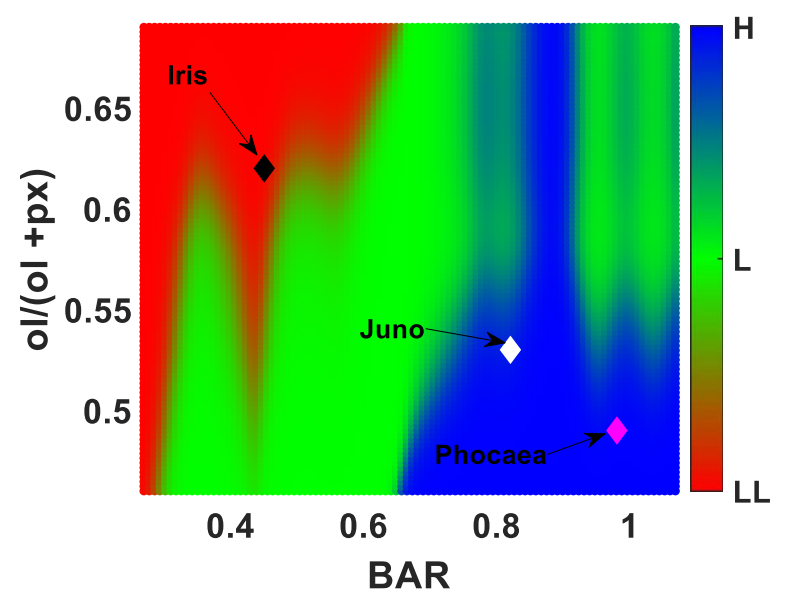

Figure 5. Color coded posteriori probability distribution evaluated by the naïve Bayesian classified $(\mathrm{H}=$ blue, $\mathrm{L}=$ green, and $\mathrm{LL}=$ red $)$ in the $(\mathrm{BAR}, \mathrm{ol} /(\mathrm{ol}$ $+\mathrm{px})$ ) space for the three main belt asteroids observed. Juno has a maximum likelihood of $89 \%$ to be $\mathrm{H}$ chondrite, and Iris has a maximum likelihood of $97.5 \%$ to be LL chondrite. The Bayes classifier shows Phocaea at a similarly high $98.6 \%$ probability of being $\mathrm{H}$ chondrite. A detailed description of the methodology as applied to asteroid spectroscopy is presented in the Appendix of Reddy et al. (2018).

Based on our Bayes classifier, Juno has a $89 \%$ probability of being $\mathrm{H}$ chondrite, $11 \%$ of being $\mathrm{L}$ chondrite, and $0 \%$ of being LL chondrite. Iris has a $97.5 \%$ probability of being an LL chondrite, and $2.5 \%$ and $0 \%$ of being $\mathrm{L}$ and $\mathrm{H}$ chondrite, respectively. Phocaea has a $98.6 \%$ probability of being $\mathrm{H}$ chondrite, $1.4 \%$ of being $\mathrm{L}$ chondrite, and $0 \%$ of being LL chondrite.

\section{Discussion}

Identifying the source of $\mathrm{H}$ chondrites is a problem that has puzzled planetary scientists for decades. Given that $\mathrm{H}$ chondrites make up 34\% of all meteorite falls (Burbine et al. 2009), one might assume that the source of the $\mathrm{H}$ chondrites would readily stand out among main belt asteroids or asteroid
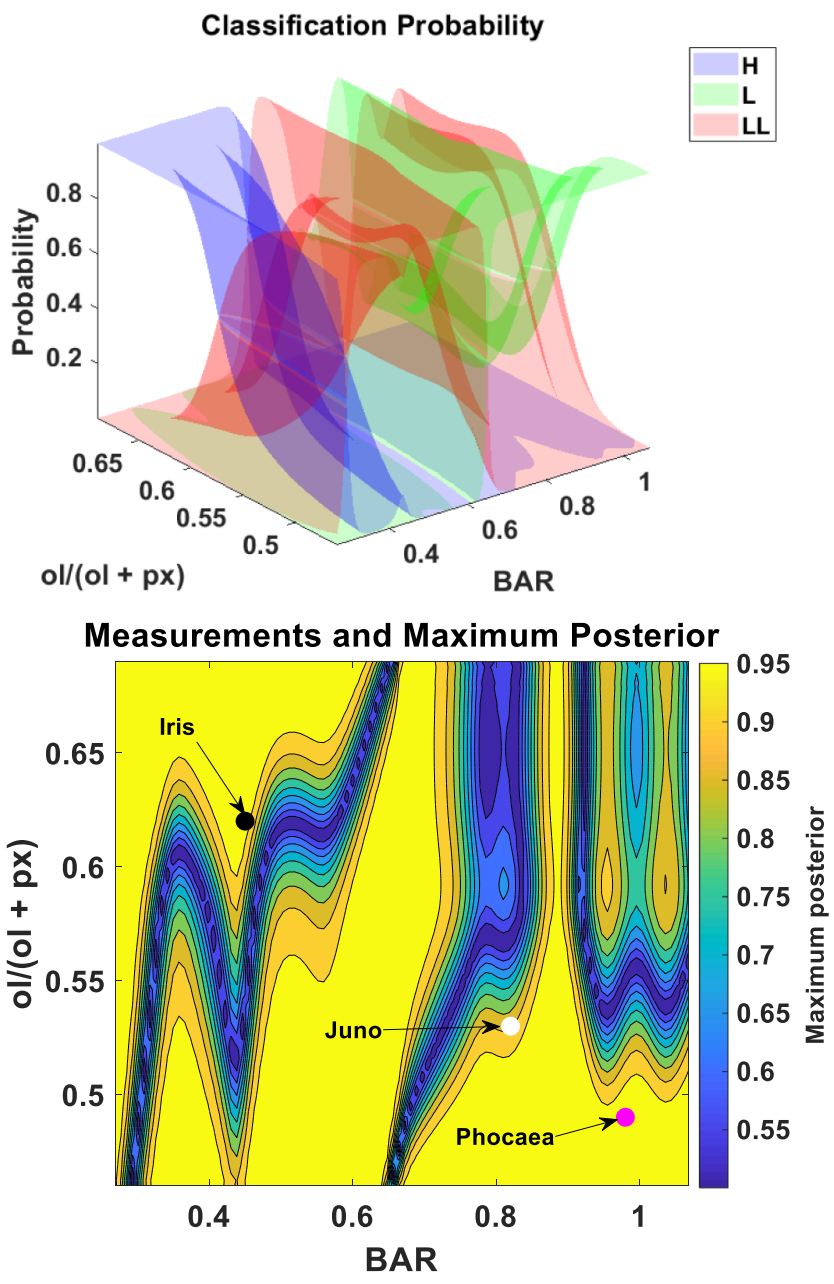

Figure 6. Posterior probability distribution as computed by the trained Bayesian classifier for the three classes $(1$ blue $=\mathrm{H}, 2$ green $=\mathrm{L}$, and 3 red $=\mathrm{LL})$. Bottom panel: contour plot for the estimated maximum posterior likelihood in the BAR and ol/(ol+px) space. In this specific plot, for each of the points in the BAR and $\mathrm{ol} /(\mathrm{ol}+\mathrm{px})$ space, the classifier computes the probability of the point to be in each of the classes and the maximum likelihood is plotted (range between 0 and 1). The position of Iris, Juno, and Phocaea in the BAR and ol $/(\mathrm{ol}+\mathrm{px})$ space is reported. Importantly, Juno has a maximum likelihood of $89 \%$ to be $\mathrm{H}$ chondrite, and Iris has a maximum likelihood of $97.5 \%$ to be LL chondrite. The Bayes classifier shows Phocaea at a similarly high $98.6 \%$ probability of being $\mathrm{H}$ chondrite. A detailed description of the methodology as applied to asteroid spectroscopy is presented in the Appendix of Reddy et al. (2018).

families. The problem is that, in general, meter-scale meteoroids are too small to be detected regularly by existing NEO surveys. Accordingly, linking a parent asteroid and/or an asteroid family to the $\mathrm{H}$ chondrites is an exercise in deduction rather than one that can be readily proved. Of the three asteroids in our study, Phocaea, has the highest probability of being an $\mathrm{H}$ chondrite $(98.6 \%)$, closely followed by Juno at $89 \%$. However, the Phocaea region appears to be a weak source of NEAs (Granvik et al. 2018; Table 5 in Granvik \& Brown 2018), and thus an unlikely source. We focus our discussion on Juno as the source body of the $\mathrm{H}$ chondrites in our discussion below, but acknowledge that Phocaea may have an H-chondrite-like surface and may not contribute significantly to the Earth's ordinary chondrites.

Given current information, there could plausibly be a single $\mathrm{H}$ chondrite parent body or several tens, with each parent body contributing to the overall flux at different levels. If we 
consider the latter, it still seems likely that one source dominates the others rather than having multiple sources contributing more or less equally. Support for this can be found in the work of Bottke et al. $(2005,2015)$, who found that the majority of meteorites are produced by a collisional cascade from an asteroid family size distribution. Their work indicates that young large families, with steeper, less ground-down size distributions, tend to produce more meteorite precursors than older smaller families. If a young prominent family is also well positioned near a good resonance for delivering things to Earth, it is possible that it can dominate all other sources. This could be the situation with the $\mathrm{H}$ chondrites. We will need additional data to make a more quantitative analysis.

In the 1990s, it was argued that (6) Hebe, was the likely source of the $\mathrm{H}$ chondrites (Farinella et al. 1993; Migliorini et al. 1997; Gaffey \& Gilbert 1998). It was favored over other candidate asteroids because: (i) Hebe had the right spectroscopic signature for $\mathrm{H}$ chondrites (e.g., $\mathrm{S}$ (IV) class), (ii) it was located near the 3:1 mean motion resonance with Jupiter and the $v 6$ secular resonance, both key transportation routes for delivering material to Earth (e.g., Morbidelli \& Gladman 1998), and (iii) Hebe is sufficiently large that cratering events could presumably inject $\mathrm{H}$ chondrites directly into these resonances.

By the 2000s, though, it became clear that despite the Yarkovsky effect being able to provide sufficient meteoroid mobility, bodies/families far from resonances may still dominate the delivery flux through a collisional cascade (e.g., Bottke et al. 2005, 2006, 2015). This has weakened evidences (ii)-(iii) as stated above. In addition, Hebe has no observed family (e.g., Nesvorný 2015), and most H-chondrites cosmicray exposure (CRE) ages are $>7 \mathrm{Ma}$ (Eugster et al. 2006), far longer than expected if meteoroids were directly injected into resonances at a steady rate (i.e., a large fraction should be $<1-2$ Ma; Morbidelli \& Gladman 1998). Moreover, recent direct imaging of (6) Hebe using Very Large Telescope/SpectroPolarimetric High-contrast Exoplanet REsearch (VLT/ SPHERE) show a paucity of large impact craters (Marsset et al. 2017). Marsset et al. (2017) argue that other families with spectra comparable to Hebe located close to the 3:1 and 5:2 mean motion resonances, namely Agnia, Koronis, Maria, Massalia, and Merxia, could potentially provide more material to the Earth than Hebe. We note that the spectral classification of the parent bodies of the families above was done by Vernazza et al. (2014).

Alternatively, Vernazza et al. $(2014,2015)$ argued that the $\mathrm{H}$ chondrites may have multiple parent bodies across the main belt. Indeed, their work showed that a multitude of large main belt asteroids and asteroid families have spectra similar to $\mathrm{H}$ chondrites as well as oxide ratios $[\mathrm{FeO} /(\mathrm{FeO}+\mathrm{MgO})]$ in olivine and pyroxene consistent with $\mathrm{H}$ chondrite meteorites. Their work also pointed out that many of these bodies have been at least partially shattered or scrambled, with highly metamorphosed H6-type materials surprisingly common on the surfaces of these bodies. The compositional links between $\mathrm{H}$ chondrites and multiple asteroids/asteroid families seems robust. Given that the Yarkovsky effect can potentially cause meteoroids from across the main belt to migrate to resonances, with some delivered to Earth, their hypothesis is not unreasonable.

The two cases above highlight the endmember situation. On the one side, we have a single well-positioned asteroid providing most $\mathrm{H}$ chondrites, while on the other, a cacophony of bodies and families are responsible. We suspect both may be right at some level. Consider that in any natural system, there may be multiple sources that contribute to a net flux, but the odds that all of these sources provide more or less equal value are usually low. Instead, one source typically dominates the others. Our interpretation of $\mathrm{H}$ chondrites constraints makes us believe this is the case here as well, with Juno as the parent body of the $\mathrm{H}$ chondrites. Consider the following.

Meteorite cosmic-ray exposure ages. Roughly half of all studied $\mathrm{H}$ chondrites have $\mathrm{CRE}$ ages of 7-8 Ma (e.g., see a review in Eugster et al. 2006). No other major meteorite class has this signature. This trait implies that a single parent body or parent family dominates other $\mathrm{H}$ chondrite sources (though other sources may still be important). The singular 7-8 Ma spike in CRE ages is short enough to suggest that the dominant $\mathrm{H}$-chondrite source is located relatively close to a major resonance that is efficient at delivering objects to Earth. Either that or we are looking at the breakup of an $\mathrm{H}$ chondrite body near or on an Earth-crossing orbit, with its contribution comparable to the background H-chondrite flux.

${ }^{40} \mathrm{Ar}-{ }^{39} \mathrm{Ar}$ shock degassing ages. Additional constraints come from the ${ }^{40} \mathrm{Ar}-{ }^{39} \mathrm{Ar}$ shock degassing ages of $\mathrm{H}$ chondrites (Swindle et al. 2014). Shock degassing ages are produced by collision events on large asteroids that yield high temperatures beyond the Ar loss threshold (i.e., cratering events with $D_{\text {crater }}>10 \mathrm{~km}$ ). Marchi et al. (2013) showed that these high temperatures were more likely to reflect impact velocities exceeding $10 \mathrm{~km} \mathrm{~s}^{-1}$. The easiest way to get such impact velocities is for bodies outside the asteroid belt, say in the planet-crossing population, to strike main belt targets.

The $\mathrm{H}$ chondrite distribution of ${ }^{40} \mathrm{Ar}-{ }^{39} \mathrm{Ar}$ ages and those found among howardite-eucrite-diogenite (HED) meteorites, presumably from Vesta, are surprisingly similar to one another for $>3.5 \mathrm{Ga}$ (e.g., Marchi et al. 2013). Both samples show a number of events $>4.4 \mathrm{Ga}$, a relative lull between 4.1 and 4.4 $\mathrm{Ga}$, and numerous events between 3.4 and $4.0 \mathrm{Ga}$. One potential explanation for these events is that both bodies saw the same impactor population and that both bodies are large, according to Bogard's rule (Scott \& Bottke 2011). Considering that energetic events occur across the asteroid belt from time to time, one would expect to see a plethora of shock ages in all meteorite types. We would also perhaps expect to see shocked material on many of the asteroids visited by spacecraft. Instead, shock degassing ages among meteorites and shock-darkened materials on asteroids are surprisingly rare. Don Bogard, for whom Bogard's Rule is named, found the abundance of samples with ${ }^{40} \mathrm{Ar}-{ }^{39} \mathrm{Ar}$ reset ages was higher among lunar samples than HEDs, and HEDs were higher than among H-type ordinary chondrites, whose parent body was likely in the $200 \mathrm{~km}$ diameter range. He suggested that the increase in the abundance of shock-reheated samples should go up with increasing parent body size, mainly because these bodies could more readily keep more of the hot ejecta than smaller bodies. Although smaller asteroidal bodies would have been preferentially destroyed during early bombardment, the proportion of shock-heated rocks on the surviving small bodies would have been lower than on the larger bodies. The corollary is that fragmental breccias are formed more efficiently on large bodies than on small ones. Based on this idea, one can argue the $\mathrm{H}$ chondrites parent body was likely one of the larger main belt bodies. 
In addition, while numerous ${ }^{40} \mathrm{Ar}-{ }^{39} \mathrm{Ar}$ ages for shocked $\mathrm{H}$-chondrites meteorites are found between 0-1 Ga, 3.5-4.1 $\mathrm{Ga}$, and 4.4-4.5 Ga, the largest concentration is found near $\sim 0.3 \mathrm{Ga}$. This may suggest something significant happened to the $\mathrm{H}$ chondrite parent body at $\sim 0.3 \mathrm{Ga}$. We speculate this event may have been a large cratering event or perhaps even a catastrophic disruption, either of which may have formed an observable family (Swindle et al. 2014). Finding context in the $\mathrm{H}$ chondrite parent body or parent family would help to make this argument less qualitative and more quantitative.

While computing an age for the Juno family by tracking the dynamical evolution of the family members (e.g., Bottke et al. 2015 ) is beyond the scope of this paper, we can estimate one as follows. (20) Massalia is a $150 \mathrm{~km}$ diameter S-type asteroid with a family produced by a large cratering event (e.g., Durda et al. 2007). Vokrouhlický et al. (2007) used Yarkovsky/ YORP dynamical evolution models to estimate that the Massalia family likely formed $150-200 \mathrm{Myr}$ ago (see also Nesvorný 2015, who list the age as $150 \pm 50 \mathrm{Myr}$ ). A proxy for the age of the Massalia family is the $\mathrm{C} 0$ parameter, which defines the dynamical envelope of an asteroid family (Nesvorný 2015). The Massalia family has $\mathrm{C} 0=0.25 \pm 0.05$, while the Juno family, also produced by a large cratering event, has $\mathrm{C} 0=0.5 \pm 0.2$. The ratio of the two values is $2 \pm 0.9$, suggesting that the Juno family is approximate twice the age of the Massalia family, or somewhere in the ballpark of 300 \pm 100 Myr. Accordingly, the age of Juno family's is consistent with the shock degassing age concentration of $\sim 0.3 \mathrm{Ga}$ seen in $\mathrm{H}$ chondrites.

Thermal modeling results. There have been many attempts to estimate the size of the $\mathrm{H}$ chondrite parent body from meteorite constraints and thermal modeling results (e.g., Ghosh et al. 2003; Trieloff et al. 2003; Amelin et al. 2005; Blinova et al. 2007; Kleine et al. 2008; Harrison \& Grimm 2010; Henke et al. 2012; Ganguly et al. 2013; Monnereau et al. 2013; Scott et al. 2014; Blackburn et al. 2017). The argument is that if parent bodies were heated by ${ }^{26} \mathrm{Al}$ and allowed to cool without impacts producing mixing, they would develop an onion-shell structure from differentiation. Here the most metamorphosed type 6 material would reside in the deeper interior, while the shells of less-metamorphosed types 5 through 3 material would surround it. We caution that the meteorite samples chosen to perform the analysis may affect the results. For example, Scott et al. (2014) argue that metallographic cooling rate data indicates that portions of the $\mathrm{H}$ chondrite parent body were shattered and scrambled early in its history. Most of these works indicate that the $\mathrm{H}$ chondrite parent body was larger than $200 \mathrm{~km}$ in diameter. The most recent works even point to the parent body being larger than $275 \mathrm{~km}$ (e.g., Blackburn et al. 2017).

Paleomagnetic evidence. Portales Valley is a metallic melt impact breccia defined as an H6 (e.g., Ruzicka \& Hugo 2018). Studies indicate the melt is $\sim 4.5 \mathrm{Ga}$ (Swindle et al. 2014) and that it has a distinct paleomagnetic signature (Bryson et al. 2016). Preliminary work indicates that the $\mathrm{H}$ chondrite parent body was generating a stable, $10 \mu \mathrm{T}$ magnetic field when the impact melt cooled below the Curie point (Bryson et al. 2016). Accretion and dynamo models aimed at constraining the possible size and accretion times of the $\mathrm{H}$ chondrite parent body using this paleomagnetic signature indicate that the $\mathrm{H}$ chondrite parent body likely had a diameter between 230 and
$320 \mathrm{~km}$, with the most likely diameter being $280 \mathrm{~km}$ (J. F. Bryson 2019, personal communication).

Taken together, these studies suggest that the $\mathrm{H}$ chondrite parent body was probably larger than $200 \mathrm{~km}$ in diameter and may potentially be associated with a relatively young family, if we are interpreting the younger ${ }^{40} \mathrm{Ar}-{ }^{39} \mathrm{Ar}$ ages for the $\mathrm{H}$ chondrite meteorites correctly. These constraints are a reasonable match to what we know about (3) Juno and its family, though we caution that we cannot yet argue we have a unique match. Juno is a $320 \times 267 \times 200 \pm 6 \mathrm{~km}$ asteroid (Baliunas et al. 2003; see also Durech et al. 2011)

Observed members of the Juno family are adjacent to the J8:3 mean motion resonance with Jupiter. This gives them, and the small meteoroids associated with them, a ready way out of the main belt. Studies of NEO and meteoroid delivery, however, indicate the J8:3 may not be powerful enough to be a strong source of asteroids or meteoroids. Juno family observed members are also close enough to the J3:1 mean motion resonance that it is likely that small unobserved meteoroids from the Juno family can reach it. That resonance is a better source of Earth impactors than the J8:3 from a probability standpoint (e.g., Bottke et al. 2006). Moreover, preliminary numerical studies indicate some Juno meteoroids jump over the J3:1 to reach the $\nu 6$ resonance (Bottke et al. 2017). A careful study of the dynamics of Juno meteoroids is an excellent project for further work. With that said, the Juno family cannot produce sizeable H-chondrite-type NEOs, even though studies suggest many H-chondrite-type NEOs come from the J3:1 (Thomas \& Binzel 2010). If Juno is the primary source of $\mathrm{H}$ chondrite meteorites, it would suggest that an additional source is needed to explain the H-chondritetype NEOs.

\section{Summary}

Infrared spectra of (3) Juno, (7) Iris, and (25) Phocaea with NASA's IRTF yielded improved measurements of BARs and olivine/(olivine+pyroxene) ratios for the targets. These ratios were compared to meteoritic samples from $\mathrm{H}, \mathrm{L}$, and LL chondrites using a Bayesian inference model to identify each asteroid's closest meteoritic analog. Based on our Bayes classifier:

1. Juno has a $89 \%$ probability of being $\mathrm{H}$ chondrite, $11 \%$ of being L chondrite, and $0 \%$ of being LL chondrite;

2. Iris has a $97.5 \%$ probability of being LL chondrite, and $2.5 \%$ and $0 \%$ of being $\mathrm{L}$ and $\mathrm{H}$ chondrite, respectively;

3. and Phocaea has a $98.6 \%$ of being $\mathrm{H}$ chondrite, $1.4 \%$ of being $\mathrm{L}$ chondrite, and $0 \%$ of being LL chondrite.

Most interesting of these identifications is the finding that the infrared spectrum of Juno most closely resembles that of the $\mathrm{H}$ chondrites, a meteorite class where roughly half of the CRE ages studied to date are 7-8 million years old. We identify several constraints in line with Juno as a plausible parent body for the $\mathrm{H}$ chondrites $\left({ }^{40} \mathrm{Ar}-{ }^{39} \mathrm{Ar}\right.$ ages, shock degassing ages, paleomagnetic measurements) and lay out future work necessary to test this hypothesis, choosing to focus on the dynamics of delivering Juno family meteoroids to Earth.

This work was supported by NASA Near-Earth Object Observations (NEOO) program grant NNXAL06G (PI: Reddy), state of Arizona Technology Research Initiative Fund (TRIF), and a generous donation from the Lunar and Planetary 
Laboratory's External Advisory Board. We thank the IRTF TAC for awarding time to this project, and to the IRTF TOs and MKSS staff for their support. The authors wish to recognize and acknowledge the significant cultural role and reverence the summit of Maunakea has always had within the indigenous Hawaiian community. We are most fortunate to have the opportunity to conduct observations from this mountain.

\section{ORCID iDs}

John W. Noonan (1) https://orcid.org/0000-0003-2152-6987

Vishnu Reddy (1) https://orcid.org/0000-0002-7743-3491

Walter M. Harris (1) https://orcid.org/0000-0002-8378-4503

Theodore Kareta ำ https://orcid.org/0000-0003-1008-7499

\section{References}

Amelin, Y., Ghosh, A., \& Rotenberg, E. 2005, GeCoA, 69, 505

Baliunas, S., Donahue, R., Rampino, M. R., et al. 2003, Icar, 163, 135

Blackburn, T., Alexander, C. M. D., Carlson, R., \& Elkins-Tanton, L. T. 2017, GeCoA, 200, 201

Blinova, A., Amelin, Y., \& Samson, C. 2007, M\&PS, 42, 1337

Bottke, W. F., Vokrouhlický, D., Walsh, K. J., et al. 2015, Icar, 247, 191

Bottke, W., Mazrouei, S., Ghent, R., \& Parker, A. 2017, AAS/DPS Meeting, 49, 100.03

Bottke, W. F., Jr, Nolan, M. C., Greenberg, R., Kolvoord, R. A., et al. 1994, Icar, 107, 255

Bottke, W. F., Jr, Durda, D. D., Nesvorný, D., et al. 2005, Icar, 175, 111

Bottke, W. F., Jr, Vokrouhlický, D., Rubincam, D. P., \& Nesvorný, D. 2006, AREPS, 34, 157

Bryson, J. F. J., Weiss, B. P., Scholl, A., et al. 2016, AGU Abstracts, 2016, P53D-02

Burbine, T. H., McCoy, T. J., Meibom, A., Gladman, B., \& Keil, K. 2009, in Asteroids III, ed. W. F. Bottke, Jr., A. Cellino, P. Paolicchi, \& R. P. Binzel (Tucson, AZ: Univ. Arizona Press), 653

Carruba, V. 2009, MNRAS, 398, 1512

Cloutis, E. A., Gaffey, M. J., Jackowski, T. L., \& Reed, K. L. 1986, JGR, 91, 11641

Conrad, A., Le Mignant, D., Dumas, C., et al. 2003, BAAS, 35, 959

Cushing, M. C., Vacca, W. D., \& Rayner, J. T. 2004, PASP, 116, 362

DeMeo, F. E., Binzel, R. P., Slivan, S. M., \& Bus, S. J. 2009, Icar, 202, 160

Dunn, T. L., McCoy, T. J., Sunshine, J. M., \& McSween, H. Y. 2010, Icar, 208, 789

Durda, D. D., Bottke, W. F., Jr., Nesvorný, D., et al. 2007, Icar, 186, 498

Durech, J., Kaasalainen, M., Herald, D., et al. 2011, Icar, 214, 652

Eugster, O., Herzog, G. F., Marti, K., \& Caffee, M. W. 2006, in Meteorites and the Early Solar System II, ed. D. S. Lauretta \& H. Y. McSween, Jr. (Tucson, AZ: Univ. Arizona Press), 829

Farinella, P., Gonczi, R., Froeschlé, C., \& Froeschlé, C. 1993, Icar, 101, 174

Gaffey, M. J., Bell, J. F., Brown, R. H., et al. 1993, Icar, 106, 573

Gaffey, M. J., \& Gilbert, S. L. 1998, M\&PS, 33, 1281

Ganguly, J., Tirone, M., Chakraborty, S., \& Domanik, K. 2013, GeCoA, 105,206

Ghosh, A., Weidenschilling, S. J., \& McSween, H. Y., Jr. 2003, M\&PS, 38,711
Granvik, M., \& Brown, P. 2018, Icar, 311, 271

Granvik, M., Morbidelli, A., Jedicke, R., et al. 2018, Icar, 312, 181

Groeneveld, I., \& Kuiper, G. P. 1954, ApJ, 120, 529

Harrison, K. P., \& Grimm, R. E. 2010, GeCoA, 74, 5410

Henke, S., Gail, H. P., Trieloff, M., Schwarz, W. H., Kleine, T., et al. 2012, A\&A, 545, A135

Kaasalainen, M., Torppa, J., \& Piironen, J. 2002, Icar, 159, 369

Kleine, T., Touboul, M., Van Orman, J. A., et al. 2008, E\&PSL, 270, 106

Magri, C., Nolan, M. C., Ostro, S. J., \& Giorgini, J. D. 2007, Icar, 186, 126

Marchi, S., Bottke, W. F., Cohen, B. A., et al. 2013, NatGe, 6, 303

Marsset, M., Carry, B., Dumas, C., et al. 2017, A\&A, 604, A64

Masiero, J. R., DeMeo, F. E., Tasuga, T., \& Parker, A. H. 2015, in Asteroids IV, ed. P. Michel, F. E. DeMeo, \& W. F. Bottke (Tucson, AZ: Univ. Arizona Press), 323

Masiero, J. R., Mainzer, A. K., Grav, T., et al. 2011, ApJ, 741, 68

Masiero, J. R., Mainzer, A. K., Grav, T., et al. 2012, ApJL, 759, L8

McGraw, A. M., Reddy, V., \& Sanchez, J. A. 2018, MNRAS, 476, 630

Migliorini, F., Zappala, V., Vio, R., \& Cellino, A. 1995, Icar, 118, 271

Migliorini, F., Morbidelli, A., Zappala, V., et al. 1997, M\&PS, 32, 903

Monnereau, M., Toplis, M. J., Baratoux, D., \& Guignard, J. 2013, GeCoA, 119,302

Morbidelli, A., \& Gladman, B. 1998, M\&PS, 33, 999

Nakamura, T., Noguchi, T., Tanaka, M., et al. 2011, Sci, 333, 1113

Nesvorný, D. 2015, Nesvorn'y HCM Asteroid Families V1.0, EARAVARGBDET-5-NESVORNYFAM-V1.0, NASA Planetary Data System

Nesvorný, D., Vokrouhlický, D., Morbidelli, A., \& Bottke, W. F. 2009, Icar, 200, 698

Novaković, B., Tsirvoulis, G., Granvik, M., \& Todović, A. 2017, AJ, 153, 266

Ostro, S. J., Magri, C., Benner, L. A. M., et al. 2010, Icar, 207, 285

Oszkiewicz, D. A., Bowell, E., Wasserman, L. H., et al. 2012, Icar, 219, 283

Pilcher, F. 2011, MPBu, 38, 76

Rayner, J. T., Toomey, D. W., Onaka, P. M., et al. 2003, PASP, 115, 362

Reddy, V., Dunn, T. L., Thomas, C. A., Moskovitz, N. A., \& Burbine, T. H. 2015, in Asteroids IV, ed. P. Michel, F. E. DeMeo, \& W. F. Bottke (Tucson, AZ: Univ. Arizona Press), 43

Reddy, V., Emery, J. P., Gaffey, M. J., et al. 2009, M\&PS, 44, 1917

Reddy, V., Sanchez, J. A., Furfaro, R., et al. 2018, AJ, 155, 8

Ruzicka, A. M., \& Hugo, R. C. 2018, GeCoA, 234, 115

Sanchez, J. A., Michelsen, R., Reddy, V., \& Nathues, A. 2013, Icar, 225, 131

Sanchez, J. A., Reddy, V., Dykhuis, M., Lindsay, S., \& Le Corre, L. 2015 , ApJ, 808, 93

Sanchez, J. A., Reddy, V., Nathues, A., et al. 2012, Icar, 220, 36

Scott, E. R. D., \& Bottke, W. F. 2011, M\&PS, 46, 1878

Scott, E. R. D., Krot, T. V., Goldstein, J. I., Wakita, S., et al. 2014, GeCoA, 136, 13

Swindle, T. D., Kring, D. A., \& Weirich, J. R. 2014, GSLSP, 378, 333

Tedesco, E. F., Cellino, A., \& Zappalá, V. 2005, AJ, 129, 2869

Thomas, C. A., \& Binzel, R. P. 2010, Icar, 205, 419

Trieloff, M., Jessberger, E. K., Herrwerth, I., et al. 2003, Natur, 422, 502

Ueda, Y., Miyamoto, M., Mikouchi, T., \& Hiroi, T. 2003, LPI, 34, 2078

Usui, F., Kuroda, D., Müller, T. G., et al. 2011, PASJ, 63, 1117

Vernazza, P., Binzel, R. P., Thomas, C. A., et al. 2008, Natur, 454, 858

Vernazza, P., Marsset, M., Beck, P., et al. 2015, ApJ, 806, 204

Vernazza, P., Zanda, B., Binzel, R. P., et al. 2014, ApJ, 791, 120

Viikinkoski, M., Kaasalainen, M., Durech, J., et al. 2015, A\&A, 581, L3

Vokrouhlický, D., Breiter, S., Nesvorný, D., \& Bottke, W. F. 2007, Icar, 191, 636

Warner, B. D., Harris, A. W., \& Pravec, P. 2009, Icar, 202, 134 